\title{
El cambio climático y la vigilancia del FMI. La necesidad de tener ambición ${ }^{1}$
}

Climate Change and IMF Surveillance. The Need for Ambition

\author{
Kevin P. Gallagher ${ }^{2}$ \\ Universidad de Boston, Estados Unidos \\ Luma Ramos $^{3}$ \\ Centro de Política de Desarrollo Global \\ Corinne Stephenson ${ }^{4}$ \\ Centro de Política de Desarrollo Global/ \\ Universidad de Boston, Estados Unidos \\ Irene Monasterolo ${ }^{5}$ \\ Universidad de Economía y Negocios de Viena, Austria/ \\ Centro de Política de Desarrollo Global
}

Revista Derechos en Acción ISSN 2525-1678/ e-ISSN 2525-1686

Año 6/No 18 Verano 2020-2021 (21 diciembre a 20 marzo), 816-832

DOI: https://doi.org/10.24215/25251678e497

1 Texto originalmente publicado como GEGI Policy Brief 014, de marzo de 2021, disponible en https://www.bu.edu/gdp/files/2021/03/GEGI_PB_014_IMF_Climate_Surveillance.pdf Traducción y adaptación a cargo de Francisco Cantamutto,

2 Profesor en la Universidad de Boston y director del Centro de Política de Desarrollo Global en la misma universidad.

3 Investigadora posdoctoral en el Centro de Política de Desarrollo Global.

4 Becaria en el Centro de Política de Desarrollo Global y estudiante de doctorado en la Universidad de Boston.

5 Profesora asistente en la Universidad de Economía y Negocios de Viena, e investigadora asociada del Centro de Política de Desarrollo Global. 
Resumen: El Fondo Monetario Internacional (FMI) necesita diseñar rápidamente una estrategia de cambio climático que ayude a sus miembros a cumplir sus objetivos colectivos de cambio climático y desarrollo. En este breve informe se describen los aspectos macrocríticos del cambio climático que deberían incorporarse a la actividad de supervisión del FMI y se examina en qué medida los riesgos climáticos han formado parte de la supervisión del FMI en los últimos años. Nuestra investigación muestra que el FMI ha prestado una atención mínima y desigual a los riesgos climáticos en los informes del Artículo IV y aún menos en sus Programas de Evaluación del Sector Financiero (PESF), aunque en ambos casos el FMI ha experimentado con análisis que pueden servir de base para trabajar. Como resultado de las revisiones del Artículo IV y los PESF en curso, es imperativo que el FMI reconozca que tanto los riesgos físicos como los de transición dentro de los países y entre ellos son amenazas macrocríticas para los sistemas financieros y fiscales; que garantice que los análisis de los riesgos físicos y de transición dentro de los países y entre ellos sea obligatorio, sistemático y universal para todos los informes del Artículo IV y PESF de aquí en adelante; y que el FMI trabaje con el personal, los Estados miembros, las partes interesadas, la Red para la Ecologización del Sistema Financiero (NGFS) y los/as expertos/as para aprovechar su experiencia inicial y alinear la política de supervisión del FMI con los ambiciosos objetivos del cambio climático.

Abstract: The International Monetary Fund (IMF) needs to rapidly devise a climate change strategy that helps its members meet their collective climate change and development goals. This policy brief outlines the macro-critical aspects of climate change that will need to be incorporated into IMF surveillance activity and examines the extent to which climate risks have been a part of IMF surveillance in recent years. Our research shows that the IMF has paid minimal and uneven attention to climate risks in Article IV reports, and even less so in its Financial Sector Assessment Programs (FSAPs) — though in each case the IMF has experimented with analyses that can be built upon. As a result of the reviews of Article IV surveillance and FSAPs currently underway, it is imperative that the IMF: recognize that both physical and transition risks within and across countries are macro-critical threats to financial and fiscal systems; ensure that physical and transitions risks analysis within and across countries is compulsory, systematic, and universal for 
all Article IV and FSAPs moving forward; and that the IMF work with staff, member states, stakeholders, the NGFS, and experts to build on its early experience and align IMF surveillance policy with ambitious climate change goals.

\section{Introducción}

El Fondo Monetario Internacional (FMI) necesita diseñar rápida pero cuidadosamente una estrategia de cambio climático que ayude a los países a cumplir sus objetivos colectivos en materia de cambio climático, de forma que se potencie la estabilidad, la equidad, el crecimiento y el desarrollo sostenible. Una de las principales prioridades de la reforma del FMI será alinear sus funciones básicas de supervisión con la ambición climática. Para ello, el FMI se comprometió recientemente a incorporar el cambio climático en sus revisiones del Artículo IV y de los Programas de Evaluación del Sector Financiero (PESF). Este informe sobre políticas describe los aspectos macrocríticos del cambio climático que deben incorporarse a la actividad de supervisión del FMI, examina hasta qué punto los riesgos climáticos han formado parte de la supervisión del FMI en los últimos años, y presenta recomendaciones para incorporar el riesgo climático en los exámenes del Artículo IV y del PESF.

La clave para prevenir y mitigar las implicaciones macrocríticas del cambio climático es la necesidad de considerar los riesgos físicos y de transición para los sistemas fiscales y financieros, tanto dentro de los países como entre ellos. Los riesgos físicos se producen cuando los efectos materiales del cambio climático, como la mayor incidencia de huracanes, dañan los activos físicos, aumentando así el riesgo crediticio y las pérdidas financieras para los inversores. Los riesgos de transición surgen de una introducción tardía y descoordinada de políticas climáticas cuyos impactos no pueden ser totalmente anticipados por los inversores, lo que lleva a ajustes repentinos de los precios 
de los activos. ${ }^{6}$ Basándonos en análisis recientes y de próxima aparición, encontramos una atención muy limitada a los riesgos climáticos en los programas de supervisión del FMI hasta la fecha. Aunque el FMI ha empezado a prestar más atención a los riesgos físicos en los informes del Artículo IV, esto se excluye en su mayoría de los PESF, con la excepción de un trabajo pionero para el FMI sobre el riesgo de transición en 2020. Estos resultados son a la vez preocupantes y alentadores. Es preocupante que el FMI haya tardado en reconocer la importancia macrocrítica del cambio climático. Pero es alentador que el FMI esté empezando a adoptar las herramientas necesarias para incorporar el riesgo climático en sus actividades de vigilancia.

Es primordial que este impulso se acelere y se integre en las actuales supervisiones. Como resultado de las revisiones actuales, el Artículo IV y los PESF deben examinar obligatoriamente los riesgos físicos y de transición, así como los que se transmiten a través de los efectos de propagación. El FMI también tendrá que disponer de notas de orientación y formación claras para garantizar que el análisis del riesgo climático se incorpore de forma coherente y transparente en todo el FMI. Por último, el FMI tendrá que trabajar con las partes interesadas, la Red para la Ecologización del Sistema Financiero (NGFS) y los/as expertos/as para garantizar que sus esfuerzos de vigilancia estén debidamente alineados con los ambiciosos objetivos climáticos y de desarrollo.

\section{La necesidad de análisis de riesgo climático en el FMI}

Está surgiendo un consenso sobre el hecho de que el cambio climático plantea graves riesgos macroeconómicos para el sistema financiero, tanto a nivel nacional como mundial. Una creciente red de bancos centrales y supervisores organizados como

6 Stefano Battiston, Antoine Mandel, Irene Monasterolo, Franziska Schütze y Gabriele Visentin, "A climate stress-test of the financial system", 4 Nature Climate Change, 2017, Vol. 7, 283-288. 
la Red para la Ecologización del Sistema Financiero (NGFS) ha aceptado el llamamiento de la comunidad científica, los gobiernos y la sociedad civil y ha comenzado a incorporar el cambio climático en sus herramientas de evaluación de riesgos. Más recientemente, el FMI ha reconocido la necesidad de introducir consideraciones climáticas en sus operaciones. ${ }^{7}$ Como principal institución multilateral encargada de garantizar la estabilidad del sistema monetario internacional, el FMI puede desempeñar un papel clave en el apoyo a estos esfuerzos nacionales desde una perspectiva global. A principios de este año, la directora gerente del FMI, Kristalina Georgieva, se comprometió a situar el cambio climático en el centro de su trabajo:

Mientras intentamos salir de la pandemia de COVID-19 y de la crisis económica que ha desencadenado, debemos enfrentarnos a una amenaza mayor: la del cambio climático. Es un riesgo fundamental para la estabilidad económica y financiera. También es una oportunidad para revitalizar el crecimiento y crear nuevos empleos verdes. Nuestra investigación muestra que la combinación de un aumento constante de los precios del carbono con un impulso de las infraestructuras verdes puede incrementar el PIB mundial en los próximos 15 años en un $0,7 \%$ aproximadamente y generar trabajo para millones de personas. Por eso, en el FMI apoyamos la transición a la nueva economía climática: una economía con bajas emisiones de carbono y resiliente al clima, que ayude a combatir las causas del cambio climático y adaptarse a sus consecuencias. $^{8}$

Además de proporcionar liquidez de emergencia y asesoramiento en materia de políticas, la supervisión es un pilar fundamental de la misión del FMI, que se lleva a cabo a nivel

7 Tobias Adrian, James Morsink y Liliana Schumacher, Assessing Climate-Change Risk by Stress Testing for Financial Resilience, Blog del FMI, (5 de febrero de 2020).

8 Kristalina Georgieva, "Remarks by IMF Managing Director at the Climate Adaptation Summit" (25 de enero de 2021). 
mundial, regional y nacional. Según el propio organismo, "el FMI identifica los riesgos potenciales para la estabilidad y recomienda los ajustes de política necesarios para sostener el crecimiento económico y promover la estabilidad financiera y económica". ' Las dos herramientas de supervisión bilateral son las consultas del Artículo IV y los Programas de Evaluación del Sector Financiero (PESF). De hecho, la directora gerente encargó al FMI que incorporara el cambio climático en el Examen de Supervisión Integral (ESI) y en los PESF. ${ }^{10}$

El ESI guiará la supervisión hasta 2030 con las siguientes prioridades: "(i) afrontar los riesgos y las incertidumbres; (ii) prevenir y mitigar los efectos secundarios; (iii) fomentar la sostenibilidad económica; y (iv) adoptar un enfoque más unificado del asesoramiento en materia de políticas". ${ }^{11} \mathrm{La}$ revisión del PESF incorporará el cambio climático en sus análisis de los aspectos de estabilidad financiera y desarrollo de los sistemas financieros y fiscales de los países miembros. Al hacerlo, el FMI tratará de incorporar los riesgos climáticos en análisis de resiliencia del sector bancario, en las pruebas de resistencia de los sistemas bancarios y en los efectos transfronterizos así como en la calidad del marco jurídico y las instituciones diseñadas para promover la estabilidad y el crecimiento. ${ }^{12}$ Este informe de políticas resume el trabajo reciente y en curso para ayudar a guiar al FMI y a las partes interesadas en el trabajo de incorporar el cambio climático en las operaciones del organismo.

En la siguiente sección de este breve informe de política se esbozan los aspectos macrocríticos de los riesgos físicos y

9 FMI, Further extension of consultation cycles due to COVID-19 Pandemic, and suspension of framework to address excessive delays in Article IV Consultations and mandatory Financial Stability Assessments, FMI, Washington (julio de 2020).

10 Georgieva, 2021, op. cit.

11 FMI, 2020, op. cit.

12 FMI, Financial Sector Assessment Program (FSAP), FMI, Washington (3 de junio de 2019). 
de transición. La sección 3 evalúa brevemente la medida en que el FMI ha abordado estas cuestiones en el pasado reciente. La última sección resume las principales conclusiones y esboza los contornos de la reforma que serán necesarios en las revisiones de la CSR y del PESF.

\section{Aspectos macrocríticos de los riesgos climáticos}

En la última media década, el análisis del riesgo climático en el sector financiero ha evolucionado considerablemente en la bibliografía académica y en los bancos centrales, y ahora está ganando terreno en el FMI. En esta sección describimos tres aspectos clave del riesgo climático que son de importancia fundamental para que el FMI incorpore en su actividad de vigilancia.

Los riesgos financieros derivados del cambio climático incluyen tanto "riesgos físicos" como "riesgos de transición". Los riesgos físicos (Figura 1) se derivan de fenómenos meteorológicos inmediatos, así como de cambios a largo plazo en el clima. Estos riesgos se caracterizan por su creciente gravedad, volatilidad y frecuencia. Son riesgos financieros porque afectan al valor de los activos financieros, como las propiedades y las infraestructuras, entre otros. A su vez, los bancos se enfrentan a mayores riesgos de crédito y de suscripción. ${ }^{13}$

\footnotetext{
13 Sandra Batten, Rhiannon Sowerbutts y Misa Tanaka, Let's Talk About the Weather: The Impact of Climate Change on Central Banks, Working Paper N ${ }^{0} 603$, Banco de Inglaterra (Londres, 2016); Emanuele Campiglio, Yannis Dafermos, Pierre Monnin, Josh Ryan-Collins, Guido Schotten y Misa Tanaka, "Climate change challenges for central banks and financial regulators", Nature Climate Change, 2018, Vol. 8, 462-468; Simone Dikau y Ulrich Volz, "Central Banking, Climate Change, and Green Finance", en Jeffrey Sachs, Wing Thye Woo, Naoyuki Yoshino y Farhad Taghizadeh-Hesary (eds.) Handbook of Green Finance, Singapore, Springer; Irene Monasterolo, "Climate change and the financial system", Annual Review of Resource Economics, 2020, Vol. 12, 1-22.
} 
Figura 1. Aspectos macrocríticos del "riesgo físico"

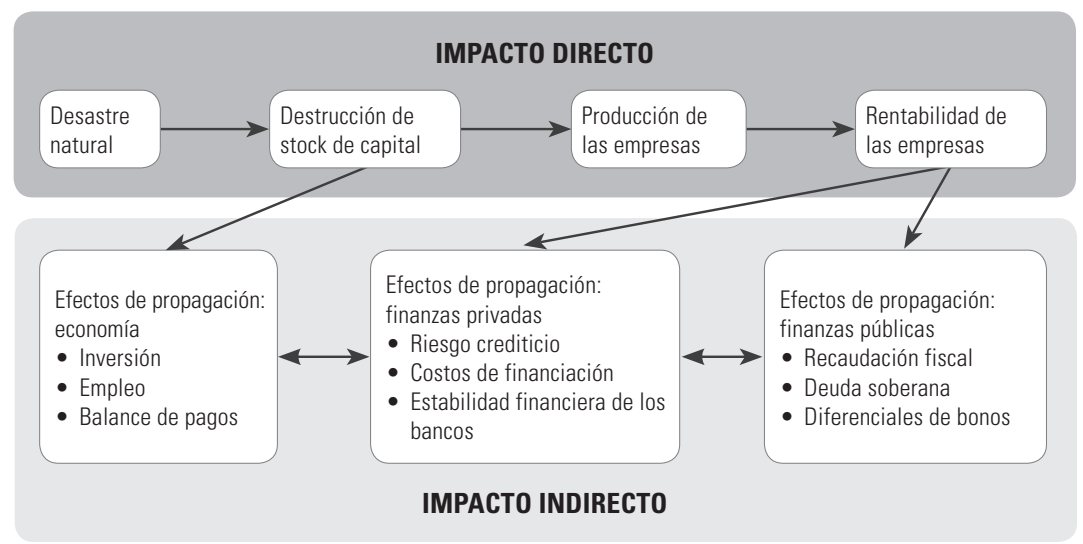

Fuente: adaptado de Dunz. ${ }^{14}$

El riesgo físico puede tener importantes repercusiones macrofinancieras en los países, que son relevantes para ser analizadas. Imagínese el caso de la Figura 1 como un Pequeño Estado Insular en Desarrollo (PEID) en el Caribe que depende en gran medida del turismo para el crecimiento, el empleo y para obtener divisas. Se ha demostrado que estos países vulnerables al clima ya tienen que pagar un mayor costo de capital por esas vulnerabilidades, creando problemas de espacio fiscal y sostenibilidad de la deuda. ${ }^{15}$ Por lo tanto, los aumentos imprevistos de la incidencia de peligros naturales, como los huracanes, pueden traducirse en un duro shock de oferta a través de la destrucción de hoteles, carreteras y transporte aéreo. Esa destrucción del stock de capital puede tener un impacto negativo y propagarse sobre los niveles de crecimiento y empleo, y en la balanza de pagos del país. Por supuesto, el nivel de producción y la rentabilidad

\footnotetext{
14 Nepomuk Dunz, Andrea Mazzocchetti, Irene Monasterolo y Marco Raberto, "The macroeconomic and financial impact of compounding COVID-19 and climate physical risk", Working paper presentado en el Seminario de Investigación 2020 del Banco Mundial, 2020.

15 Gerhard Kling, Ulrich Volz, Victor Murinde y Sibel Ayas, "The Impact of Climate Vulnerability on Firms' Cost of Capital and Access to Finance", World Development, 2021, Vol. 137, 1-11.
} 
disminuyen en las empresas afectadas, lo que puede tener efectos negativos en el sistema financiero y, a través de pérdidas en los ingresos fiscales, en los diferenciales de los bonos, etc.

Los riesgos de transición (figura 2) son los que conlleva el proceso de transición a una economía con bajas emisiones de carbono a nivel nacional. Esto genera una amplia gama de regulaciones basadas en la cantidad y el precio, como la prohibición de las centrales eléctricas de carbón, los impuestos al carbón, y esquemas que incrementan los costos de producción y reducen la rentabilidad de las empresas intensivas en el uso de carbón, lo que causa que algunos activos queden "varados" y otros ganen valor. Existe un riesgo adicional de responsabilidad, que se refiere a los riesgos legales de las partes afectadas negativamente por el cambio climático. ${ }^{16}$

Figura 2. Aspectos macrocríticos del riesgo de propagación de transición

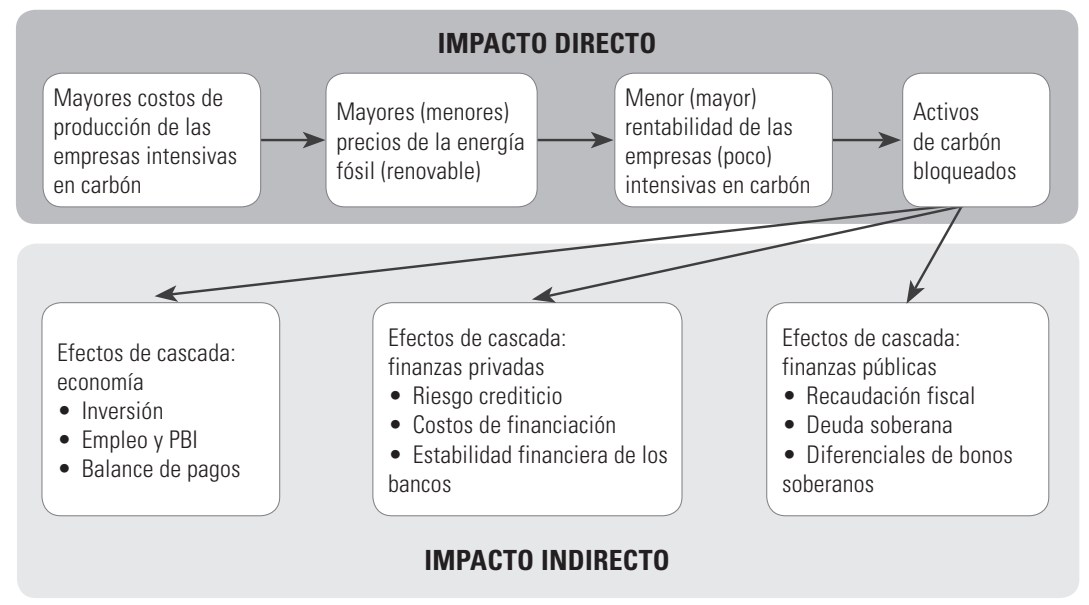

Fuente: adaptado de Monasterolo. ${ }^{17}$

16 Frank Ackerman, Worst-Case Economics: Extreme Events in Climate and Finance, Anthem Press, Londres, 2017; Battiston et al., 2017, op. cit.; Dikau y Volz, 2019, op. cit.; Monasterolo, 2020, op. cit.

17 Irene Monasterolo, "Climate change and the financial system" Annual Review of Resource Economics, 2020, Volume 12, 1-22. 
El abandono del carbón se considera el primer paso más importante en la transición al carbono en todo el mundo. Siguiendo la figura 2, es posible imaginarse a Polonia o Sudáfrica, dos países en los que el porcentaje de producción de electricidad a partir del carbón es de más del $60 \%$, y que ponen en marcha un importante impuesto sobre el carbono o la eliminación gradual de las centrales eléctricas de carbón. Estas medidas también suponen grandes riesgos para la economía real y para los sistemas fiscales y financieros. Por definición, un impuesto sobre el carbono aumentaría los costos de producción y los precios de las centrales eléctricas de carbón, además reduciría su rentabilidad, hasta el punto que se considerarían activos de carbono bloqueados, una tendencia que ya se está extendiendo por todo el mundo. ${ }^{18}$

De hecho, los riesgos de transición son quizás los más macrocríticos por sus posibles impactos en la economía real y las condiciones de vida, los sistemas financieros y las finanzas públicas. La figura 2 describe algunos de los efectos indirectos de propagación que se producen, como el deterioro del empleo y el crecimiento, la balanza de pagos (para los países que exportan carbón), la estabilidad financiera, los ingresos fiscales y el riesgo soberano. Aunque la exposición a todos los tipos de combustibles fósiles es cada vez más arriesgada, varios bancos centrales ven riesgos de transición debidos a la extracción de carbón y al cierre de centrales eléctricas de carbón como la forma más macrocrítica de riesgo climático, dada la profundidad de dicha exposición y el consenso de que el carbón debería ser la primera fuente de energía de la cual diversificarse. ${ }^{19}$ El Banco de

18 Ben Caldecott, Stranded Assets and the Environment: Risk, Resilience and Opportunity, Routledge, Londres, 2018; Suphi Sen y Marie-Theres von Schickfus, "Climate policy, stranded assets, and investors' expectations", Journal of Environmental Economics and Management, 2020, Vol. 100, 1-43.

19 Robert Vermeulen, Edo Schets, Melanie Lohuis, Barbara Kölbl, David-Jan Jansen y WiIlem Heeringa, The Heat Is on: A Framework for Measuring Financial Stress Under Disruptive Energy Transition Scenarios, DNB Working Paper Nº 625, De Nederlandsche Bank (2019); 
Inglaterra estima que una rápida transición del carbón podría dar lugar a reducciones de capital de entre el 40 y el $65 \%$ en la extracción y la generación de carbón, respectivamente. ${ }^{20}$

Los primeros trabajos sobre el riesgo climático y la estabilidad financiera fueron de carácter nacional, dado su origen en la comunidad de bancos centrales. Como institución mundial, el FMI se encarga de supervisar los efectos de propagación transfronterizos. Para ello, además de los riesgos de transición a nivel nacional, estamos desarrollando el concepto de "riesgos de propagación de transición”, según el cual los riesgos físicos o de transición que se producen en un país o región tienen efectos macroeconómicos transfronterizos en los sistemas financieros y los fiscales. ${ }^{21}$

Es posible imaginarse a la Unión Europea poniendo en marcha un gran impuesto sobre el carbono con un mecanismo de ajuste en frontera, tal como se ilustra en la Figura 3. Pero la perspectiva cambia observando desde la perspectiva de un país en desarrollo que depende en gran medida de exportar petróleo o gas (ya sea en forma de crudo o indirectamente a través del turismo) como Angola, Azerbaiyán Congo, Ecuador, México, Timor Oriental y los países en desarrollo del Golfo Pérsico. En esos casos una crisis del precio del petróleo o del gas reduce las exportaciones y tiene un impacto inmediato en la balanza de pagos.

\footnotetext{
Thomas Allen, Stephane Dees, Jean Boissinot, Carlos Mateo Caicedo, Valérie Chouard, Laurent Clerc, Annabelle de Gaye, Antoine Devulder, Sébastien Diot, Noëmie Lisack, Fulvio Pegoraro, Marie Rabaté, Romain Svartzman y Lucas Vernet, Climate-Related Scenarios for Financial Stability Assessment: An Application to France, Working Paper Series 774, Bank of France (2020).

20 Nathaniel Bullard, "Climate change puts insurers to the test", Bloomberg, 29 de junio de 2019.

21 Luma Ramos, Corinne Stephenson, Irene Monasterolo y Kevin Gallagher, "Climate Risk and IMF Surveillance Policy: A Baseline Analysis", Global Development Policy Center Working Paper, Boston, (2021); Irene Monasterolo y Kevin Gallagher, Modeling the Impacts of Climate Change Induced Transition Spillovers in Developing Countries: a Macro-financial Approach, mimeo, Global Development Policy Center Working Paper (Boston, 2021).
} 
Figura 3. Aspectos macrocríticos de la propagación del riesgo de transición

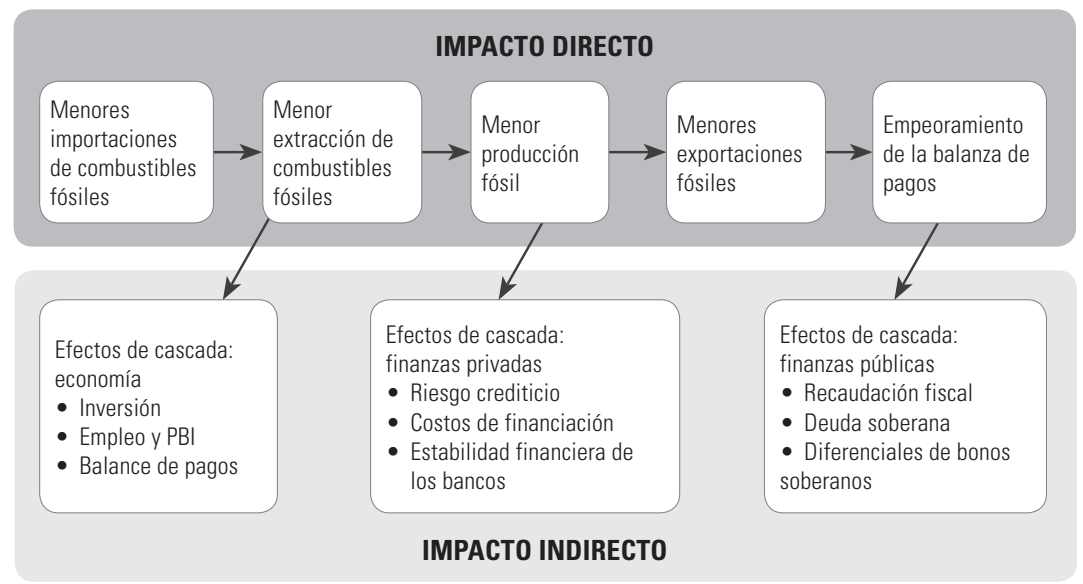

Fuente: Adaptado de Monasterolo y Gallagher, 2021.

De hecho, fue un shock como este lo que provocó muchas de las crisis financieras de la década de 1980, y que siguen siendo inesperadas y desencadenan inestabilidad. ${ }^{22}$ Los efectos de propagación de la transición climática no solo desencadenarán shocks en la balanza de pagos, sino que también pueden llegar en cascada a la economía real, a las finanzas privadas y, por supuesto, a las finanzas públicas, especialmente en las economías dependientes de los combustibles fósiles.

\section{Los esfuerzos de vigilancia no prestan suficiente atención a los riesgos climáticos}

Hasta ahora el FMI no ha llevado a cabo sus actividades de supervisión prestando atención al riesgo climático. En un análisis de los informes del Artículo IV y de los PESF desde 2017 hasta el presente, encontramos que, con algunas excepciones

22 Christiane Baumeister y Lutz Kilian, "Forty Years of Oil Price Fluctuations: Why the Price of Oil May Still Surprise Us", 1 Journal of Economic Perspectives, 2016, Vol. 30, 139-60. 
prometedoras, el FMI aún no ha incorporado el análisis del riesgo climático en estos programas, especialmente en los países que pueden enfrentar los mayores riesgos climáticos.

Tabla 1. Conteo de términos seleccionados en revisiones de Artículo IV y PESF

\begin{tabular}{|l|c|c|c|c|c|c|c|c|}
\hline & \multicolumn{4}{|c|}{ Artículo IV } & \multicolumn{5}{c|}{ PESF } \\
\cline { 2 - 10 } & 2017 & 2018 & $\mathbf{2 0 1 9}$ & $\mathbf{2 0 2 0}$ & $\mathbf{2 0 1 7}$ & $\mathbf{2 0 1 8}$ & $\mathbf{2 0 1 9}$ & 2020 \\
\hline Riesgo climático & 7 & 2 & 15 & 3 & 0 & 0 & 0 & 5 \\
\hline Riesgo físico & 0 & 0 & 0 & 0 & 0 & 0 & 0 & 4 \\
\hline Riesgo de transición & 3 & 3 & 2 & 3 & 2 & 0 & 1 & 15 \\
\hline
\end{tabular}

Fuente: Gallagher et al., 2021 (nota 18).

Desde 2017 el FMI ha realizado y publicado 384 informes del Artículo IV y 66 PESF centrados en la evaluación de riesgos. Como se muestra en la tabla 1, aunque muchos de estos informes buscan discutir el cambio climático con cierto detalle, todavía tienen que llevar a cabo la supervisión a través de las mismas lentes que la comunidad de bancos centrales. El término "riesgo climático" ha cobrado cierta importancia en los informes del Artículo IV, pero mucho menos en los PESF. El riesgo de transición tampoco ha figurado en gran medida en estos informes, salvo en un alentador PESF piloto sobre Noruega en 2020. En la supervisión del FMI no se ha mencionado el riesgo climático de propagación hasta el momento.

Si bien la tabla 1 presenta un panorama sombrío de la atención prestada por el FMI al riesgo climático, hay un margen de matiz para el optimismo. Basándose en Volz (2020) y en Volz y Ahmed (2020), ${ }^{23}$ en un análisis textual más exhaustivo de toda la vigilancia bilateral del FMI desde 2017 hasta el 1 de marzo

23 Ulrich Volz, Climate-proofing the Global Financial Safety Net, mimeo, SOAS University of London (Londres, 2020); Ulrich Volz y Sara Ahmed, Macrofinancial Risks in Climate Vulnerable Developing Countries and the Role of the IMF - Towards a Joint V20-IMF Action Agenda, SOAS Centre for Sustainable Finance, Global Center on Adaptation, and Munich Climate Insurance Initiative (Londres, Rotterdam y Bonn, 2020). 
de 2021, creamos un corpus de nomenclatura relacionada con el clima y los riesgos climáticos y adoptamos un algoritmo para revisar estos documentos y calibrar la atención del FMI al cambio climático y al riesgo relacionado.

A través de ese ejercicio creamos un "Índice de Vigilancia Climática del FMI", como la relación entre la suma de los términos de búsqueda por grupo y la frecuencia inversa del documento como porcentaje, de 0 a 1 . Aquí, una puntuación más alta significa una mayor atención al cambio climático por publicación para cada país miembro de la muestra. En ese análisis encontramos poca atención al cambio climático, pero sí encontramos que el FMI ha prestado cierta atención al riesgo físico para los Estados más pequeños en los informes del Artículo IV $\mathrm{y}$ ha puesto a prueba un prometedor PESF en Noruega. ${ }^{24}$

Los informes del Artículo IV del FMI han prestado poca atención a los riesgos físicos en los países más vulnerables al riesgo climático en la economía mundial. ${ }^{25}$ Níger, Somalia, Chad, Benín, Myanmar, Liberia y Uganda son países muy vulnerables al riesgo climático físico, según el índice de vulnerabilidad ND-Gain (que mide la vulnerabilidad climática en forma de riesgos físicos como huracanes, sequías, etc., para los países de todo el mundo según las mediciones de Chen et al., 2015). Sin embargo, en 2019, en el índice de vigilancia climática tuvieron puntuaciones bajas o cero puntuaciones, lo que indica una escasa atención a los riesgos físicos en los países más impactados por el cambio climático. En nuestro análisis más amplio, sin embargo, encontramos que el $55 \%$ de toda la atención al cambio climático en los informes del Artículo IV se produce en los Estados pequeños de todo el mundo. ${ }^{26}$

\footnotetext{
24 Gallagher et al., 2021, op. cit.

25 El documento original incluye gráficos con las estimaciones aquí comentadas, que aquí omitimos en aras de brevedad. El mismo se encuentra disponible en https://www.bu.edu/ gdp/files/2021/03/GEGI_PB_014_IMF_Climate_Surveillance.pdf
}

26 Gallagher et al., 2021, op. cit. 
Una historia similar se presenta con respecto a los riesgos de transición, especialmente en el sector del carbón. El FMI no tiene en cuenta los riesgos relacionados con los activos de carbono bloqueados en ninguno de los países, excepto en dos (Mongolia y Botsuana, donde la proporción de electricidad procedente de centrales eléctricas de carbón es muy elevada). Sin embargo, la investigación relacionada con Mongolia muestra que el FMI ha dado asesoramiento inconsistente a Mongolia en las consultas del Artículo IV de ese país: por un lado, reconoce la dependencia del carbón para ese país, pero también lo alienta a crear mejores incentivos de inversión para los depósitos de carbón del país. ${ }^{27}$

Aunque los riesgos de propagación son una parte importante de las actividades de supervisión del FMI, no hay pruebas de que el FMI esté considerando estos efectos indirectos de la transición en sus análisis hasta la fecha. Considerando el Índice de Vigilancia del Clima del FMI de 2019 con relación a las exportaciones de combustibles fósiles como porcentaje de las exportaciones totales, se encuentra una relación totalmente negativa, ya que el FMI presta menos atención al cambio climático cuanto más expuesto está un país a los mercados mundiales de combustibles fósiles.

En este informe de políticas no incluimos las conclusiones de nuestros documentos con respecto a los PESF. Nuestra investigación encuentra que no se ha prestado atención al cambio climático en los PESF, salvo en un caso. Entre 2017 y 2021 hubo 66 PESF relevantes que solo mencionaron el cambio climático 127 veces -y 105 de esas menciones fueron en un solo PESF pionero sobre Noruega-. Como parte del PESF de Noruega, el FMI llevó a cabo un análisis de los shocks de riesgo de transición nacionales en las empresas noruegas y también modeló los canales de riesgo de transición a través de un escenario

27 Recourse, IMF in Mozambique and Mongolia: Exacerbating climate crisis with more tax breaks for coal and gas, Amsterdam, 2020. 
en el que se produce un aumento significativo en los precios mundiales del carbono que repercute en Noruega a través del sector del petróleo. ${ }^{28}$

\section{Sumario y recomendaciones para el Examen de Supervisión Integral y los PESF}

Este breve informe de políticas resume los estudios pioneros y la práctica de los bancos centrales que ha surgido para abordar los aspectos macroeconómicos del cambio climático. El cambio climático es macrocrítico para las economías nacionales y la economía mundial en general a través de los riesgos físicos y de transición. Y lo que es más importante, demostramos cómo algunos riesgos de transición pueden transmitirse a través de las fronteras, a lo que nos referimos como riesgos de propagación de transición.

También resumimos los trabajos recientes y futuros sobre la medida en que el FMI ha incorporado los riesgos físicos y de transición en las actividades de supervisión del Artículo IV y del PESF desde 2017. Encontramos que el FMI ha prestado poca y desigual atención a los riesgos climáticos en los informes del Artículo IV, y aún menos en PESF. De hecho, identificamos análisis prometedores de los riesgos físicos en algunos Estados pequeños en las recientes consultas del Artículo IV, y un análisis piloto del riesgo de transición para un PESF en Noruega.

Es primordial que el FMI amplíe rápidamente esta experiencia para alinear todas sus funciones con ambiciosos objetivos climáticos. El Examen de Supervisión Integral y la revisión del PESF son las primeras oportunidades concretas para hacerlo. Dado el tiempo que transcurre entre las revisiones de supervisión y la urgencia del problema climático, es imperativo que las revisiones del Examen de Supervisión Integral y de los PESF de 2021

28 Pierpaolo Grippa y Samuel Mann, Climate-Related Stress Testing: Transition Risks in Norway, International Monetary Fund Working Paper. WP/20/232, Washington, 2020. 
den lugar a directivas que reconozcan que los riesgos físicos y de transición son igualmente macrocríticos y que requieren:

- la evaluación de los riesgos físicos y de transición para todos los países en las Consultas del Artículo IV y los Programas de Evaluación del Sector Financiero de forma obligatoria, sistemática y universal;

- la evaluación de los riesgos indirectos de transición como parte de las consultas del Artículo IV y de los PESF que también se incorporan a los esfuerzos de supervisión multinacional y a los informes sobre efectos indirectos; y

- serán necesarias notas directrices y capacitaciones específicas para ayudar al personal de vigilancia a identificar los canales macrocríticos para los riesgos físicos y de transición y a realizar el análisis necesario para ayudar a los Estados miembros a identificar y mitigar los riesgos.

Durante este año crucial, es esencial que el FMI trabaje con los Estados miembros, las partes interesadas, la NGFS y los/as académicos/as para aprovechar su experiencia inicial y alinear de forma ambiciosa la política de vigilancia del FMI con los objetivos del cambio climático. 